\title{
Peri-implant tissue alteration around tissue-level and bone-level implants in fresh extraction sockets: a histomorphometric study in dogs
}

\author{
Chunan Zhang ${ }^{1 \#}$, Xu Zhao ${ }^{2 \#}$, Shichong Qiao ${ }^{1}$, Xiaomeng Zhang ${ }^{1}$, Hongchang Lai $^{1}$, Yingxin Gu ${ }^{1}$ \\ ${ }^{1}$ Department of Implant Dentistry, Shanghai Ninth People's Hospital, College of Stomatology, Shanghai Jiao Tong University School of Medicine, \\ National Clinical Research Center for Oral Diseases, Shanghai Key Laboratory of Stomatology \& Shanghai Research Institute of Stomatology, \\ Shanghai, China; ${ }^{2}$ Peking University Hospital of Stomatology first division, Beijing, China \\ Contributions: (I) Conception and design: Y Gu, C Zhang; (II) Administrative support: H Lai; (III) Provision of study materials or patients: X Zhao; (IV) \\ Collection and assembly of data: S Qiao; (V) Data analysis and interpretation: X Zhang; (VI) Manuscript writing: All authors; (VII) Final approval of \\ manuscript: All authors. \\ "These authors contributed equally to this work. \\ Correspondence to: Yingxin Gu. Department of Implant Dentistry, Shanghai Ninth People's Hospital, Shanghai Jiao Tong University School of \\ Medicine, 639 Zhizaoju Road, Shanghai 200011, China. Email: yingxingu@163.com.
}

Background: To compare tissue alteration in fresh extraction sockets between bone-level and tissue-level implants with different neck designs.

Methods: Bilateral premolars of 6 adult Labrador dogs were extracted, and 24 bone-level and tissuelevel implants with two different neck designs were immediately placed. At the same time, buccal bony wall thickness in fresh extraction sockets was also recorded. The Straumann® Bone Level (BL) and Standard Plus (SP) implants were positioned at two insertion depths: $1 \mathrm{~mm}$ below and flush with the alveolar crest. All animals were sacrificed 6 months after the implant placement. Undecalcified block sections were obtained for histological measurement. Vertical bone resorption and biological widths were documented. Statistical analysis consisted of two sample t-test and Wilcoxon sign-rank test.

Results: All implants were histologically osseointegrated. There was no significant difference between BL implants and SP implants in vertical bone resorption regardless of the insertion depths $(\mathrm{P}>0.05)$. Meanwhile, significant difference was found in lingual biological width between BL $(3.16 \mathrm{~mm})$ and SP $(2.43 \mathrm{~mm})$ implants when placed $1 \mathrm{~mm}$ below the alveolar crest $(\mathrm{P}<0.05)$.

Conclusions: Within the limits, it seemed that different implant neck designs had little effect on bone remodeling in fresh extraction sockets. However, longer biological width was found in bone-level implants.

Keywords: Tissue alteration; tissue-level implants; bone-level implants; fresh extraction sockets

Submitted Nov 24, 2020. Accepted for publication Jan 21, 2021.

doi: 10.21037/atm-20-8074

View this article at: http://dx.doi.org/10.21037/atm-20-8074

\section{Introduction}

Over the last three decades, immediate implant placement in fresh extraction sockets has become a treatment option in daily practice and numerous short- and long-term clinical studies have demonstrated that excellent implant survival rates could be achieved with its use. However, studies have also shown that immediate implant placement failed to prevent bone and soft tissue resorption (1).

Research has suggested that along with the neck surface and type of connection between the implant and the prosthetic, implant neck design can influence surrounding tissue when implants are placed in fresh extraction sockets in clinical trial and dog model (2-4). Tissue-level implants with an expanded neck have been widely used in clinical 
practice and while marginal bone loss is considered to be acceptable in healed alveolar ridges, the expanded implant neck configuration can lead to crater-shaped bone resorption. However, data on implants designed with a smooth expanded coronal collar placed in fresh extraction sockets remains scarce. Platform switching design has been considered to be effective in reducing bone resorption (5). However, whether this design would minimize bone resorption after immediate implant placement remains unclear. On the other hand, there is little information on soft tissue remodeling using different neck designs. Thus, more studies are needed to compare the effects on tissue remodeling between bone-level implants with platform switching design and tissue-level implants with smooth expanded neck in fresh extraction sockets.

The relationship between implant insertion depth and crest of the alveolar ridge has also been shown to have certain influence on tissue alteration. Some studies have proposed that implants should be placed below the buccal alveolar crest so as to counteract alveolar bone resorption $(6,7)$. Moreover, variation in insertion depths may also exert some effect on biological width establishment. To date, only a few studies have explored the impact of insertion depth with bone-level and tissue-level implants on biological width establishment in fresh extraction sockets (8).

Therefore, the purpose of our study was to compare tissue alteration in fresh extraction sockets between bonelevel and tissue-level implants with different neck designs. We speculate that compared to tissue-level implants with divergent smooth collars, bone-level implants with platform switching might help reduce tissue resorption in immediate implant placement. We present the following article in accordance with the ARRIVE reporting checklist (available at http://dx.doi.org/10.21037/atm-20-8074).

\section{Methods}

\section{Implant placement}

Six male Labrador dogs (21-22 kg) with a mean age of one year were selected for this study. The study was approved by the Animal Care and Experiment Committee of the hospital affiliated to the Shanghai Ninth People's Hospital, Shanghai Jiao Tong University, School of Medicine and Complied with the National Institutes of Health Guide for the Care and use of Laboratory animals (NIH Publications No. 8023, revised 1978). All efforts were made to minimize animal suffering and to reduce the number of animals used following the European Commission Directive 86/609/ EEC for animal experiments. All animals were kept in kennel cages at the hospital animal research center and provided with appropriate veterinary care and free access to water and nutritional support throughout the study.

All dogs were pre-anesthetized with ketamine hydrochloride injection $(10 \mathrm{mg} / \mathrm{kg}$ i.m.) and procaine (procaine $40 \mathrm{mg}$, adrenaline $0.05 \mathrm{mg}$, local anesthesia). During surgery, full thickness flaps were elevated to expose the buccal and lingual bony walls. The mandibular premolars selected for extraction bilaterally were then split in a buccal-lingual direction at the bifurcation to extract each root individually. All extractions were carefully performed to keep the remaining bony plates intact. The widths of the buccal bony wall were measured with a vernier caliper at four different depths: the top edge of the buccal wall and 1, 3, and $5 \mathrm{~mm}$ below the crest. As the first premolar extraction site was considered too small for implant, only the remaining premolar sites were used.

Straumann ${ }^{\circledR}$ Bone Level (BL) and Standard Plus (SP) implants with SLA (sand-blasted large grit acid-etched) surface (Straumann AG, Basel, Switzerland) were used. The SP implants were soft tissue level implants with a turned shoulder with a neck diameter of $4.8 \mathrm{~mm}$, and the height of the turned surface was $1.8 \mathrm{~mm}$. The BL implants used were platform switching implants with a horizontal offset of $0.265 \mathrm{~mm}$. The neck of BL implants was micro-thread design. All SP and BL implants used had a diameter of $3.3 \mathrm{~mm}$ and length of $10 \mathrm{~mm}$. All implant placements were performed by a surgeon with 5 years of experience in clinical practice. For surgery, same 2.2 and $2.8 \mathrm{~mm}$ drills were used for both BL and SP implants.

A total of 24 implants were placed with each dog receiving four implants which included two SP implants and two BL implants. These were randomly placed immediately in the six extraction sockets (Figure 1, Table 1). The SP implants were placed either crestally or $1 \mathrm{~mm}$ subcrestally with reference to the SLA surface and the BL implants were placed at $0 \mathrm{~mm}$ (the shoulder of the implant flush with the alveolar crest), and $-1 \mathrm{~mm}$ (1 mm below the alveolar crest). The healing abutments were screwed onto the implants. The size of the healing caps was $5.5 \mathrm{~mm} \times$ $2 \mathrm{~mm}$ for tissue level implants and $3.6 \mathrm{~mm} \times 3.5 \mathrm{~mm}$ for bone level implants, and the wound margins were stabilized with 4-0 Vicryl absorbable sutures (Johnson \& Johnson, Ethicon, Livingston, UK). For both BL and SP implants, flaps were sutured to allow a non-submerged healing.

Antibiotic prophylaxis of penicillin (800,000 U/day) was 

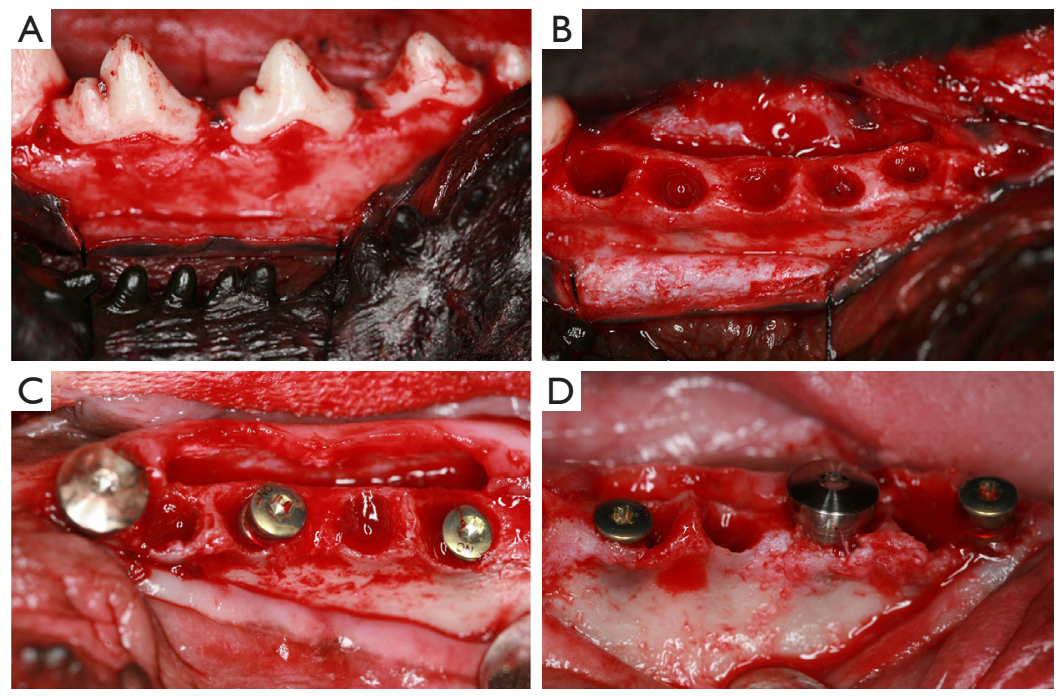

Figure 1 Surgical procedures used in the experiment. (A) The full mucoperiosteum has been elevated to the buccal side to expose the four premolars; (B) all the roots of the mandibular premolars have been separated and extracted and the buccal bony wall was intact; (C) the different types of implant were inserted into the sockets in different depth according to the plan; (D) a thin buccal wall can be seen.

administered to the dogs during the first 3 days following the surgery. Plaque control and hygiene practice were maintained once per week during the healing period under general anesthesia and the uncovered implant healing caps were cleaned with a toothbrush.

All implants healed without prosthetic appliance and specimens were harvested after 6 months of total healing time.

\section{Histological preparation}

The specimens containing implants and surrounding soft and hard tissue were fixed in $10 \%$ formalin solution. All specimens were dehydrated with increasing alcohol concentration from $50 \%$ to $100 \%$ then infiltrated in a glycol-methacrylate resin. Specimens were then sectioned in the buccal-lingual direction using the slicing system (Leica SP1600 Saw Microtome, Leica, Germany) and all implants were sectioned through the center of the implant screw. Subsequently, specimens were fixated to the Plexiglas and ground to the final thickness of about $70 \mu \mathrm{m}$.

Sections were stained by the Van Gieson staining method and observed under an Olympus BX 51 microscope (Tokyo, Japan). All images were then captured using an Olympus DP 71 digital camera and transferred to the computer. Landmarks were identified and marked on the images on both buccal and lingual sides.

Perpendicular lines were made parallel to the longitudinal axis of the implant and the following linear measurements were calculated through image processing software (ImagePro Plus Version 6.0.0.260) (Figure 2): (PM) free mucosal margin; (IS) implant shoulder; (BIC) most coronal boneto-implant contact point; $(\mathrm{aBE})$ most apical border of the barrier junctional epithelium. The following distances were then measured:

(I) PM-aBE, the length of the epithelia;

(II) aBE-BIC, the length of the connective tissue;

(III) BW: the biologic width was equal to the sum of $\mathrm{PM}-\mathrm{aBE}$ and aBE-BIC;

(IV) IS-BIC: the vertical bone resorption was also measured as the distance of IS-BIC minus the predesigned insertion depth. For SP implants, the additional $1.8 \mathrm{~mm}$ was deducted.

\section{Statistical analysis}

Statistical analysis was completed using the SPSS statistics program (IBM SPSS statistics version 19.00, IBM, USA) and reviewed by an independent statistician. A descriptive statistic was taken for each of the variables including means, standard deviations, and medians. Values are presented as mean $\pm \mathrm{SD}$ (standard deviations) and median. 
Table 1 Randomization scheme for implant location and experimental groups

\begin{tabular}{lcccccc}
\hline Dog & Site 1 & Site 2 & Site 3 & Site 4 & Site 5 & Site 6 \\
\hline 1 & C & & & D & B & A \\
2 & & D & A & C & B & \\
3 & & & C & D & A & B \\
4 & D & & & B & A & C \\
5 & B & & & C & A & D \\
6 & B & A & & C & D & \\
\hline
\end{tabular}

A: SP implants $0 \mathrm{~mm}$; B: SP implants $-1 \mathrm{~mm}$; C: BL implants 0 $\mathrm{mm}$; D: BL implants $-1 \mathrm{~mm}$.

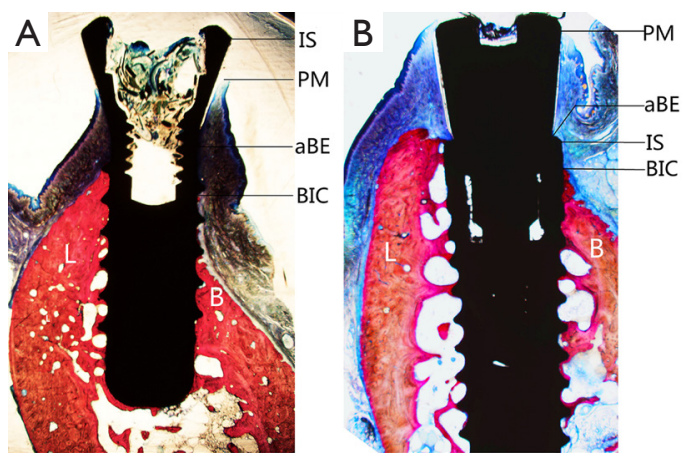

Figure 2 Histological views (Van Gieson stain) of crestal bone changes at implants with different neck design. (A) The landmarks shown on the sample of Straumann Standard Plus (SP) implant (original magnification $12.5 \times$ ). (B) The landmarks shown on the sample of Straumann bone level (BL) implant (original magnification $12.5 \times$ ). IS, implant shoulder; PM, peri-implant mucosal margin; BIC, first bone-to-implant contact point; aBE, apical end of the barrier epithelium; L, lingual; B, buccal.

The Levene homogeneity of variance test was performed before the comparison and the two sample $t$-test was used to compare the differences between implant types and insertion depths when the $\mathrm{P}$ value of the Levene homogeneity of variance test did not reach the significance level. When it did, the non-parametric Wilcoxon signrank test was performed. The level of significance was set at $\alpha=0.05$ for all statistical analysis.

\section{Results}

\section{Clinical observations}

Healing was uneventful. The mucosa adjacent to the
Table 2 Buccal bone width of the canine models at tip, $1 \mathrm{~mm}$, $3 \mathrm{~mm}$, and $5 \mathrm{~mm}$ apically to the alveolar ridge

\begin{tabular}{lcccc}
\hline \multirow{2}{*}{ Depth } & \multicolumn{4}{c}{ Sites } \\
\cline { 2 - 5 } & $\mathrm{A}(\mathrm{mm})$ & $\mathrm{B}(\mathrm{mm})$ & $\mathrm{C}(\mathrm{mm})$ & $\mathrm{D}(\mathrm{mm})$ \\
\hline 0 & $0.53 \pm 0.16$ & $0.68 \pm 0.22$ & $0.63 \pm 0.16$ & $0.71 \pm 0.19$ \\
-1 & $0.78 \pm 0.13$ & $0.93 \pm 0.26$ & $0.77 \pm 0.21$ & $0.90 \pm 0.21$ \\
-3 & $1.28 \pm 0.30$ & $1.29 \pm 0.42$ & $1.20 \pm 0.39$ & $1.23 \pm 0.33$ \\
-5 & $1.54 \pm 0.49$ & $1.58 \pm 0.55$ & $1.50 \pm 0.41$ & $1.57 \pm 0.40$ \\
\hline
\end{tabular}

implants appeared to be relatively healthy without suppuration or visible inflammation and all implants were osseointegrated. The widths of the buccal bone plate at different depths are presented in Table 2 and no significant differences were found among the four sites.

\section{Comparison between SP and BL implants}

Table 3 shows the alteration of vertical bone height and biologic width around SP and BL implants. The number of each unit was six.

When the implants were placed flush with the buccal bone crest, the mean loss of buccal bone height was $3.15 \pm 0.52 \mathrm{~mm}$ in the SP group and $2.70 \pm 0.52 \mathrm{~mm}$ in the BL group while the lingual bone loss was $1.68 \pm 0.38 \mathrm{~mm}$ in the SP group and $1.68 \pm 0.37 \mathrm{~mm}$ in the BL group. When the implants were placed $1 \mathrm{~mm}$ below the buccal bone crest, the mean loss of buccal bone height was $2.55 \pm 0.41 \mathrm{~mm}$ in the SP group and $2.99 \pm 0.21 \mathrm{~mm}$ in the BL group, while the lingual bone loss was $1.47 \pm 0.18 \mathrm{~mm}$ in the SP group and $1.56 \pm 0.19 \mathrm{~mm}$ in the $\mathrm{BL}$ group. No significant difference was found between the two groups regardless of the insertion depth.

When the implants were placed flush with the buccal bone crest, the mean buccal biologic widths were $3.34 \pm 0.66 \mathrm{~mm}$ in the SP group and $3.44 \pm 1.11 \mathrm{~mm}$ in the BL group, while the lingual biologic widths were $2.43 \pm 0.25 \mathrm{~mm}$ in the SP group and $3.00 \pm 0.58 \mathrm{~mm}$ in the $\mathrm{BL}$ group. When the implants were placed $1 \mathrm{~mm}$ below the buccal bone crest, the mean buccal biologic widths were $3.35 \pm 0.42 \mathrm{~mm}$ in the SP group and $3.57 \pm 0.57 \mathrm{~mm}$ in the BL group, while the lingual biologic widths were $2.43 \pm 0.42 \mathrm{~mm}$ in the SP group and $3.16 \pm 0.36 \mathrm{~mm}$ in the BL group. A statistically significant difference of biologic widths was found only at the lingual side when the implants were placed $1 \mathrm{~mm}$ below the buccal alveolar ridge $(\mathrm{P}=0.009)$. 
Table 3 The alteration of soft and hard tissue around SP and BL implants: mean \pm SD

\begin{tabular}{|c|c|c|c|c|c|c|}
\hline Variable & \multicolumn{3}{|c|}{ Insertion depth=0 (mm) } & \multicolumn{3}{|c|}{ Insertion depth=-1 (mm) } \\
\hline Buccal resorption & $3.15 \pm 0.52$ & $2.70 \pm 0.52$ & 0.165 & $2.55 \pm 0.41$ & $2.99 \pm 0.21$ & 0.091 \\
\hline Lingual resorption & $1.68 \pm 0.38$ & $1.68 \pm 0.37$ & 1.000 & $1.47 \pm 0.18$ & $1.56 \pm 0.19$ & 0.419 \\
\hline Buccal biological width & $3.34 \pm 0.66$ & $3.44 \pm 1.11$ & 0.853 & $3.35 \pm 0.42$ & $3.57 \pm 0.57$ & 0.464 \\
\hline
\end{tabular}

*, significant difference between SP and BL implants; Bone resorption, the distance of IS-BIC minus the pre-design insertion depth; Biological width, the distance of the sum of PM-aBE and aBE-BIC. SP, Straumann standard plus; BL, Straumann bone level.

Table 4 The alteration of soft and hard tissue around SP implants at different depth: mean \pm SD

\begin{tabular}{|c|c|c|c|c|c|c|}
\hline Variable & Buccal resorption & Lingual resorption & $P$ value & Buccalbiological width & Linugalbiological width & $P$ value \\
\hline Insertion depth $=-1(\mathrm{~mm})$ & $2.55 \pm 0.41$ & $1.47 \pm 0.18$ & $0.000^{*}$ & $3.35 \pm 0.42$ & $2.43 \pm 0.42$ & $0.004^{*}$ \\
\hline$P$ value & 0.510 & 0.249 & & 0.976 & 1.000 & \\
\hline
\end{tabular}

Bone resorption, the distance of IS-BIC minus the pre-design insertion depth; Biological width, the distance of the sum of PM-aBE and aBE-BIC. *, significant difference between buccal and lingual sides of implants.

Table 5 The alteration of soft and hard tissue around BL implants at different depth: mean \pm SD

\begin{tabular}{|c|c|c|c|c|c|c|}
\hline Variable & Buccal resorption & Lingual resorption & $P$ value & Buccalbiological width & Linugalbiological width & $P$ value \\
\hline Insertion depth $=-1(\mathrm{~mm})$ & $2.99 \pm 0.21$ & $1.56 \pm 0.19$ & $0.000^{*}$ & $3.57 \pm 0.57$ & $3.16 \pm 0.36$ & 0.167 \\
\hline$P$ value & 0.234 & 0.496 & & 0.804 & 0.579 & \\
\hline
\end{tabular}

Bone resorption, the distance of IS-BIC minus the pre-design insertion depth; Biological width, the distance of the sum of PM-aBE and aBE-BIC. *, significant difference between buccal and lingual sides of implants.

\section{Comparison among different depths}

Table 4 shows the alteration of vertical bone resorption and biologic width around SP implants at different depths. The buccal bone loss was $3.15 \pm 0.52$ and $2.55 \pm 0.41 \mathrm{~mm}$ at the depth of 0 and $-1 \mathrm{~mm}$, respectively and the lingual bone loss was $1.68 \pm 0.38$ and $1.47 \pm 0.18 \mathrm{~mm}$ at the depth of 0 and $-1 \mathrm{~mm}$, respectively. The buccal biologic widths were $3.34 \pm 0.66$ and $3.35 \pm 0.42 \mathrm{~mm}$ at the depth of 0 and $-1 \mathrm{~mm}$, respectively and the lingual biologic widths were $2.43 \pm 0.25$ and $2.43 \pm 0.42 \mathrm{~mm}$ at the depth of 0 and $-1 \mathrm{~mm}$, respectively.

Table 5 shows the alteration of vertical bone resorption and biologic width around BL implants at different depths. The buccal bone loss was $2.70 \pm 0.52$ and $2.99 \pm 0.21 \mathrm{~mm}$ at the depth of 0 and $-1 \mathrm{~mm}$, respectively and the lingual bone loss was $1.68 \pm 0.37$ and $1.56 \pm 0.19 \mathrm{~mm}$ at the depth of 0 and $-1 \mathrm{~mm}$, respectively. The buccal biologic widths were $3.44 \pm 1.11$ and $3.57 \pm 0.57 \mathrm{~mm}$ at the depth of 0 and $-1 \mathrm{~mm}$, respectively and the lingual biologic widths were $3.00 \pm 0.58$ and $3.16 \pm 0.36 \mathrm{~mm}$ at the depth of 0 and $-1 \mathrm{~mm}$, respectively.

\section{Discussion}

This study demonstrated that when using two different insertion depths, SP implants with an expanded smooth collar design showed comparable bone loss to BL implants with a platform switching design. However, longer lingual biological width was found in bone-level implants. Thus, our postulated hypothesis was partially accepted.

The buccal and lingual alveolar crests underwent 

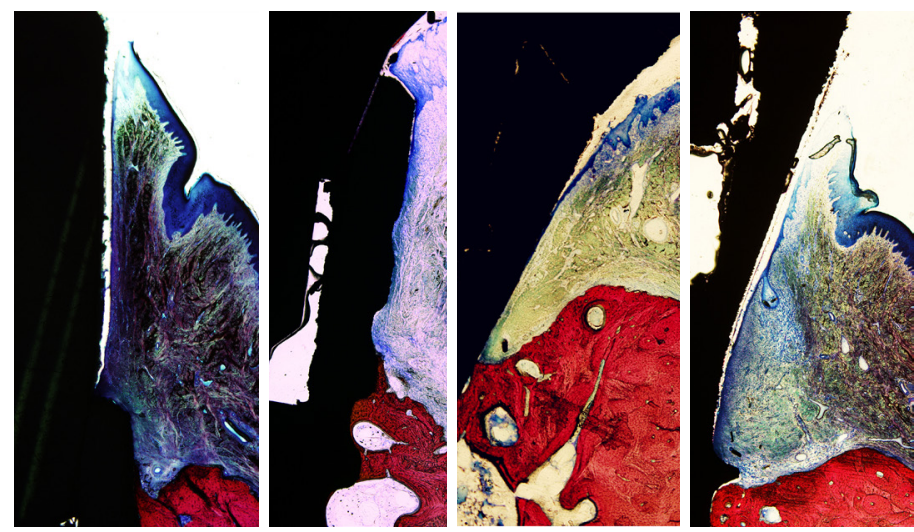

Figure 3 Lingual aspect of the soft tissue level implants and bone level implants at different depths. Notice the position of the most coronal bone to implant contact point. The most coronal bone to implant contact points for soft tissue implants were attached below the machinedrough junction interface and the most coronal bone to implant contact points for bone level implants were attached below the implantabutment junction interface. (Van Gieson stain, original magnifications 40x). From left to right: BL implant was placed 1 mm below the crestal bone level of the buccal bone; BL implant was placed at the crestal bone level of the buccal bone; SP implant was placed $1 \mathrm{~mm}$ below the crestal bone level of the buccal bone; SP implant was placed at the crestal bone level of the buccal bone.

resorption in both SP and BL implants and vertical bone resorption was more pronounced at the buccal side of the implants. This finding is in line with those published in previous animal studies $(9,10)$. As shown in Figures 2 and 3, the buccal and lingual bone levels of SP implants were both positioned below the border of the SLA surface and machined neck and the bone tissue of BL implants was attached below the edge of the implant platform or positioned just at the edge of the implant platform. These findings are in accord with the results of various experimental animal studies $(6,7,11)$. Furthermore, similar buccal and lingual change in alveolar bone level was found between SP and BL implants with platform switching design regardless of insertion depths. This might indicate that these two neck designs in immediate implant placement do not affect vertical bone resorption in fresh extraction sockets.

The results of the present study differ from those of the animal study conducted by Bressan et al. in 2014 (12) and clinical prospective study conducted by Saito et al. in 2018 (13). In the former, platform switching prototype titanium cylindrical implants with a tapered indentation of $3 \mathrm{~mm}$ long between the turned neck surface and the threads of the moderately rough surface were used. However, in our study, platform switching was located between the healing cap and implants with micro-thread design. In addition, the width of platform switching was larger in both the Bressan and Saito studies than in ours. In the Bressan study, mismatches of platform switching in test implants were 0.4 and $0.65 \mathrm{~mm}$ at the premolar and molar sites, respectively. In the Saito study, Superline implants were used and the extent of implant-abutment mismatching (0.33 and $0.58 \mathrm{~mm}$ ) were still larger than the implants used in our study, which was only $0.265 \mathrm{~mm}$. The marginal bone level around implants in tissue slides in our study were similar to those shown in other animal study where the degree of implant-abutment mismatching was $0.25 \mathrm{~mm}$ (7). Therefore, the relatively small degree of implant/abutment mismatching could be one possible reason to explain why no preservation of the socket bony crest in a more coronal level was seen. Moreover, the buccal bone was relatively thin in our study while in Bressan's study, the buccal bone width of molar sites was much thicker. The width of the original buccal bone is an important factor that will affect remodeling of the alveolar ridge after tooth extraction and immediate implant placement. As shown in Figure 4, at the coronal part of the slide, scattered bone-to-implant contact zones were found between threads of the implant by histological observation. This phenomenon indicated that when the original bone width was thin, after loss of the original buccal bone, the advanced bone resorption and apical migration of soft tissue would occur even after the initial bone-to-implant contact was completed. Finally, the observation period was only 6 months without functional loading. Therefore, a longer period with occlusal contact may produce different results. 


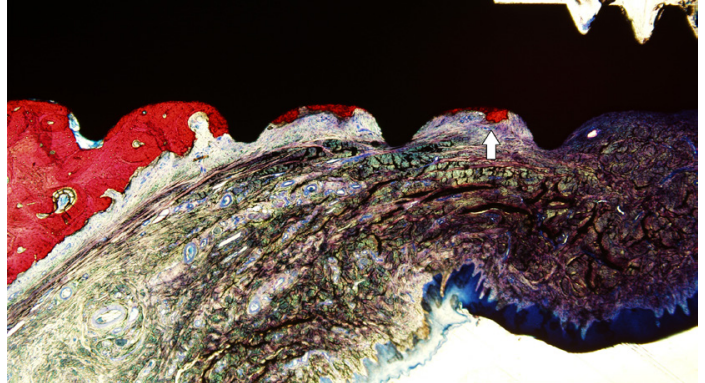

Figure 4 Scattered B-I-C zones between the threads of the implant (white arrow) were found by histological observation (Van Gieson Stain, original magnifications $40 \times$ ).

The biological widths in our study were different from those seen in previous studies $(14,15)$ which showed buccal biological widths were generally larger than lingual biological widths, although not reaching statistical significance. However, in our study, a statistically significant difference between buccal and lingual biological widths was found in SP implants $(\mathrm{P}<0.05)$. As shown by Souza et al., the design of the transmucosal component could influence the establishment of the peri-implant biological width (16). The use of implants with a divergent smooth collar in our study may have accounted for much shorter lingual biological width seen in SP implants. Compensatory soft tissue growth after buccal bone resorption was also observed in our study as it has in others (17). Thus, longer buccal biological width in SP implants could be anticipated with regards to the high proliferate rate of soft tissue compensation. However, as smooth collar was expanded, buccal soft tissue volume might not be substantial. Thus, considering aesthetic effects, the use of SP implants with tranmucosal collar design should be considered with caution.

On the other hand, it is interesting to note that in our study, biological widths of BL implants were longer than the SP implants, especially at lingual side. Longer biological widths are not only more esthetic but also have a higher volume of extracellular matrix, collagen, and increased vascularity, which might favor the immune response (18). Therefore, BL implants might be more advantageous especially in esthetic areas (19). Moreover, as stated by Grassi et al. in their recent randomized clinical trial, no-graft surgery in post-extraction immediate implant placement achieved comparable buccal bone changes to bone grafting sites (8). Thus, it could be assumed BL implants along with the no-graft design in our study might be applicable and also cost-effective in clinical situation, especially in posterior area.

The influence of insertion depths on vertical bone loss was also evaluated. Some researchers have suggested that platform switching implants should be placed sub-crestally to minimize bone loss (9). Conversely, others believe that the sub-crestal placement of implants may cause a greater amount of bone loss during the healing process than those placed in an epi or supra-crestal position (20). In our study, we found no difference between $0 \mathrm{~mm}$ and $1 \mathrm{~mm}$ subcrestal placement with reference to the SLA surface. However, from a clinical point of view, implants positioned $1 \mathrm{~mm}$ deep into extraction sockets could reduce the exposure of the neck portion of the implant. Thus, to eliminate exposure without an increase in bone resorption in esthetic areas, $1 \mathrm{~mm}$ subcrestal immediate implant placement is applicable, which is in accordance with other experimental studies (21).

Our study has several limitations. The sample size is small and only two types of implant neck design in immediate implant placement were investigated. Thus, a larger sample size study is needed for verification. Moreover, these findings resulted from a 6 -month observation and longer follow-up is needed to further validate the treatment results.

\section{Conclusions}

In conclusion, different neck designs in this study appeared to have limited influence on peri-implant bone remodeling in fresh extraction sockets. However, longer biological width was found in bone-level implants.

\section{Acknowledgments}

Funding: This study was supported by the Interdisciplinary Program of Shanghai Jiao Tong University (Project Number YG2015MS10 \& JYJC202004). The abutments and implants in the current study were supported by the Straumann Group, Basel, Switzerland.

\section{Footnote}

Reporting Checklist: The authors have completed the ARRIVE reporting checklist. Available at http://dx.doi.org/10.21037/ atm-20-8074

Data Sharing Statement: Available at http://dx.doi. 
org/10.21037/atm-20-8074

Conflicts of Interest: All authors have completed the ICMJE uniform disclosure form (available at http://dx.doi. org/10.21037/atm-20-8074). The authors have no conflicts of interest to declare.

Ethical Statement: The authors are accountable for all aspects of the work in ensuring that questions related to the accuracy or integrity of any part of the work are appropriately investigated and resolved. The study was approved by the Animal Care and Experiment Committee of the Hospital Affiliated to the Shanghai Ninth People's Hospital, Shanghai Jiao Tong University, School of Medicine and Complied with the National Institutes of Health Guide for the Care and Use of Laboratory Animals (NIH Publications No. 8023, revised 1978).

Open Access Statement: This is an Open Access article distributed in accordance with the Creative Commons Attribution-NonCommercial-NoDerivs 4.0 International License (CC BY-NC-ND 4.0), which permits the noncommercial replication and distribution of the article with the strict proviso that no changes or edits are made and the original work is properly cited (including links to both the formal publication through the relevant DOI and the license). See: https://creativecommons.org/licenses/by-nc-nd/4.0/.

\section{References}

1. Blanco J, Linares A, Perez J, et al. Ridge alterations following flapless immediate implant placement with or without immediate loading. Part II: a histometric study in the Beagle dog. J Clin Periodontol 2011;38:762-70.

2. Caneva M, Botticelli D, Rossi F, et al. Influence of implants with different sizes and configurations installed immediately into extraction sockets on peri-implant hard and soft tissues: an experimental study in dogs. Clin Oral Implants Res 2012;23:396-401.

3. Wenzel BA, Gamborena I, Lee J, et al. Effect of platform shift on crestal bone levels and mucosal profile following flap surgery and subcrestal implant placement in presence/ absence of gap defects. Clin Implant Dent Relat Res 2016;18:217-25.

4. Tan WC, Lang NP, Schmidlin K, et al. The effect of different implant neck configurations on soft and hard tissue healing: a randomized-controlled clinical trial. Clin Oral Implants Res 2011;22:14-9.
5. Telleman G, Raghoebar GM, Vissink A, et al. Impact of platform switching on inter-proximal bone levels around short implants in the posterior region; 1 -year results from a randomized clinical trial. J Clin Periodontol 2012;39:688-97.

6. Cochran DL, Mau LP, Higginbottom FL, et al. Soft and hard tissue histologic dimensions around dental implants in the canine restored with smaller-diameter abutments: a paradigm shift in peri-implant biology. Int J Oral Maxillofac Implants 2013;28:494-502.

7. Farronato D, Santoro G, Canullo L, et al. Establishment of the epithelial attachment and connective tissue adaptation to implants installed under the concept of "platform switching": a histologic study in minipigs. Clin Oral Implants Res 2012;23:90-4.

8. Grassi FR, Grassi R, Rapone B, et al. Dimensional changes of buccal bone plate in immediate implants inserted through open flap, open flap and bone grafting and flapless techniques: A cone-beam computed tomography randomized controlled clinical trial. Clin Oral Implants Res 2019;30:1155-64.

9. Calvo-Guirado JL, Moreno GG, Aguilar-Salvatierra A, et al. Bone remodeling at implants with different configurations and placed immediately at different depth into extraction sockets. Experimental study in dogs. Clin Oral Implants Res 2015;26:507-15.

10. Lee J, Fiorini T, Gamborena I, et al. Effect of platform shift/switch on crestal bone levels and mucosal profile following flapless surgery and crestal/subcrestal implant placement. Clin Implant Dent Relat Res 2016;18:73-81.

11. Alves CC, Munoz F, Cantalapiedra A, et al. Marginal bone and soft tissue behavior following platform switching abutment connection/disconnection--a dog model study. Clin Oral Implants Res 2015;26:983-91.

12. Bressan E, Lang NP, Corazza B, et al. The platform switching concept revisited. An experimental study in dogs. Clin Oral Implants Res 2014;25:1200-6.

13. Saito H, Chu SJ, Zamzok J, et al. Flapless postextraction socket implant placement: the effects of a platform switchdesigned implant on peri-implant soft tissue thickness-a prospective study. Int J Periodontics Restorative Dent 2018;38:s9-15.

14. Calvo-Guirado JL, López-López PJ, Maté Sánchez de Val JE, et al. Influence of collar design on peri-implant tissue healing around immediate implants: a pilot study in Foxhound dogs. Clin Oral Implants Res 2015;26:851-7.

15. Negri B, López Marí M, Maté Sánchez de Val JE, et al. Biological width formation to immediate implants 
placed at different level in relation to the crestal bone: an experimental study in dogs. Clin Oral Implants Res 2015;26:788-98.

16. Souza AB, Alshihri A, Kämmerer PW, et al. Histological and micro-CT analysis of peri-implant soft and hard tissue healing on implants with different healing abutments configurations. Clin Oral Implants Res 2018;29:1007-15.

17. Chappuis V, Araújo MG, Buser D. Clinical relevance of dimensional bone and soft tissue alterations post-extraction in esthetic sites. Periodontol 2000 2017;73:73-83.

18. Chappuis V, Engel O, Shahim K, et al. Soft tissue alterations in esthetic postextraction sites: a 3-dimensional analysis. J Dent Res 2015;94:187S-93S.

19. Valente NA, Wu M, Toti P, et al. Impact of concave/

Cite this article as: Zhang $\mathrm{C}$, Zhao $\mathrm{X}$, Qiao S, Zhang $\mathrm{X}$, Lai H, Gu Y. Peri-implant tissue alteration around tissuelevel and bone-level implants in fresh extraction sockets: a histomorphometric study in dogs. Ann Transl Med 2021;9(4):335. doi: $10.21037 / \mathrm{atm}-20-8074$ convergent vs parallel/ divergent implant transmucosal profiles on hard and soft peri-implant tissues: a systematic review with meta-analyses. Int J Prosthodont 2020;33:553-64.

20. Schwarz F, Mihatovic I, Golubovich V, et al. Impact of abutment microstructure and insertion depth on crestal bone changes at nonsubmerged titanium implants with platform switch. Clin Oral Implants Res 2015;26:287-92.

21. Hermann JS, Jones AA, Bakaeen LG, et al. Influence of a machined collar on crestal bone changes around titanium implants: a histometricstudy in the canine mandible. J Periodontol 2011;82:1329-38.

(English Language Editor: B. Draper) 